\title{
Drought Hazard Mapping from Regional Climate Multimodel Ensemble over Spain
}

\author{
Sandra G. García Galiano ${ }^{1,2}$, Juan Diego Giraldo-Osorio ${ }^{3}$ \& Patricia Olmos Gimenez ${ }^{1}$ \\ ${ }^{1}$ Universidad Politécnica de Cartagena, Department of Civil Engineering, R\&D Group of Water Resources \\ Management, Cartagena, Spain \\ ${ }^{2}$ University of California, Irvine, CA, USA \\ ${ }^{3}$ Pontificia Universidad Javeriana, Bogota, Colombia \\ Correspondence: Sandra G. García Galiano, Universidad Politécnica de Cartagena, Department of Civil \\ Engineering, R\&D Group of Water Resources Management, Paseo Alfonso XIII, 52, 30203, Cartagena, Spain. \\ Tel: 3496-8325-935. E-mail: sandra.garcia@upct.es
}

Received: September 3, 2015

doi:10.5539/mas.v10n2p17
Accepted: November 25, 2015 Online Published: December 24, 2015

URL: http://dx.doi.org/10.5539/mas.v10n2p17

The research is financed by National Secretary of Research of Spanish Ministry of Economy and Competitiveness (MINECO) and FEDER funds Grant No. CGL2012-39895-C02-0, and Spanish Ministry of Education, Culture and Sport for Mobility Grant of Senior Professors and Researchers Grant No. PRX14/00748.

\begin{abstract}
Improving the knowledge about the impacts of climate change on extreme drought events at basin scale, is important for decision makers in order to develop drought contingency plans which are the leading edge of adaptive management strategy. Considering high-resolution grids of observed daily rainfall and information provided by latest-generation Regional Climate Models (RCMs), the changes in the spatio-temporal patterns of extreme droughts in peninsular Spain are assessed. The non-stationarity character of time series, due to climate and anthropogenic changes, is represented by probabilistic models considering the time evolution of probability density function (PDF) parameters fitted to annual maximum lengths of dry spells time series. By a PDF ensemble from 17 RCMs, the spatio-temporal variability exhibited by the RCMs is represented. Scoring of models is based in the goodness-of-fit to CDFs (cumulative distribution functions) of observed annual maximum dry spells lengths. The reliability and skills of RCMs are assessed, for building the PDF ensemble, at grid site for the study area. Therefore, by adjusting PDF to series of annual maximum dry spells lengths, applying GAMLSS and bootstrapping techniques, the assessment of regional changes and trends associated to high returns periods ( $T r=25$ and $50 \mathrm{yr}$.) is assessed. In general, an intensification of drought events for 2050 horizon, in contrast with 1990, is expected. By increasing return periods, the length of the annual maximum dry spells rises, albeit with a smaller number of areas with significant differences. The areas prone to extreme droughts in mainland Spain are identified.
\end{abstract}

Keywords: drought hazard mapping, multimodel ensemble, climate change, GAMLSS, Spain

\section{Introduction}

Europe is regularly affected by severe water scarcity and drought events. Southern and south-eastern Europe is most prone to an increase in drought hazard, due to high demands of water. Consequently, mitigation actions and restrictions are applied on water uses, when an extreme event occurs. According to EEA (2010), due to water scarcity, physical modifications (dams, reservoirs, hydropower projects) and poor water quality, many European water bodies are at risk of failing to meet the aim of the EU Water Framework Directive to achieve good status by 2015 . In southern Spain, agriculture accounts for more than $80 \%$ of the total national abstraction. In this sense, the plausible trends in the frequency and intensity of maximum drought events, and therefore its potential impact on water availability, should be known.

There are several approaches in the analysis of drought variability. Several authors (Bonaccorso, Bordi, Cancelliere, Rossi, \& Sutera, 2003; Raziei, Saghafian, Paulo, Pereira, \& Bordi, 2008; Vasiliades, Loukas, \& Liberis, 2011) have applied those based on standardized indexes of several variables (rainfall, soil wetness, etc.). 
Then, the Mann-Kendall test was used to detect trends and cycles of drought time series (Ruíz Sinoga, García Marín, Martínez Murillo, \& Gabarron Galeote, 2011), or even applying stationary probabilistic distribution functions (PDF) for estimating extreme dry-spell risk (Vicente Serrano \& Beguería Portugués, 2003).

In the present work, the analysis of extreme droughts is assessed by spatial and temporal patterns of annual maximum dry spells lengths, corresponding to maximum consecutive days without rainfall, or with rainfall below a threshold. Annual maximum dry spells lengths have major impacts on agriculture due to their influence on soil moisture content for low return periods $(T r)$. Plausible trends of annual maximum dry spells lengths and identification of areas prone to extreme droughts are relevant for decision making in planning and management of water resources at basin scale for medium and high $T r$.

Dynamical downscaling, such as Regional Climate Models (RCMs), provides information with adequate spatial-temporal resolution for impact studies at basin scale on water resources management issues (such as floods and droughts). Global climate models (GCMs) are not suitable to produce the fine-scale climate projections needed to assess impacts at regional scale.

Since hydrological systems are affected by several drivers (land-use change, groundwater development, dam building, climate variability and change), today it is no longer possible to consider them as stationary (Milly et al., 2008). Therefore, considering these premises for building spatial distribution of annual maximum dry spells lengths associated to $T r$, the GAMLSS (Generalized Additive Models for Location, Shape and Scale) tools proposed by Rigby and Stasinopoulos (2005), are applied. GAMLSS correspond to non-stationary probabilistic modelling, being applied to extreme peak flows and maximum annual daily rainfall analysis by several authors (Villarini et al., 2009).

In the present paper, $\mathrm{CDF}$ (cumulative distribution functions) of annual maximum dry spells lengths obtained from both RCMs and observed data, are considered for the bias analysis to evaluate the ability of RCMs to represent present-day extreme droughts events. An ensemble of models is considered a more accurate description of the limitations, uncertainties and a probabilistic approach for future climate projections (Sánchez, Romera, Gaertner, Gallardo, \& Castro, 2009). Based on bias analysis, greater weight was given to the RCMs with better performance in the PDF ensemble for each site using bootstrapping techniques (Efron \& Tibshirani, 1993). Finally, from the spatial patterns of plausible annual maximum dry spells lenghts associated with several $T r$, and the time frame selected, quantitative predictions of climate change impacts on extreme drought risk at basin scale are assessed.

\section{Characterization of Study Area and Datasets}

The study area corresponding to peninsular Spain (Figure 1), presents a significant Northwest-Southeast gradient of rainfall frequency and amount, with characteristic Atlantic and Mediterranean precipitation regimes, respectively. Fourteen basins are identified in mainland Spain (Figure 1). Some of these are in the North and North-West (Euskadi basins, Miño basin, Galicia coast basins, and Cantabrian basins). Other basins drain into the Mediterranean Sea (Catalonian basins, Ebro basin, Jucar basin, Segura basin and Andalusian Mediterranean basins). Finally, basins in the South-West drain to the Atlantic Ocean (Andalusian Atlantic basins and Guadalquivir basin), and transboundary basins (Guadiana basin, Tajo basin and Duero basin).

A regular daily gridded rainfall dataset of $0.2^{\circ}$ (approx. $20 \mathrm{~km}$ ) horizontal resolution spanning the period from 1950 to 2007, named Spain02/v2.1 (or Spain02) is used, derived from a very dense network of 2756 quality-controlled stations (Herrera, Fita, Fernández, \& Gutiérrez, 2010).

The results provided by seventeen latest-generation RCMs driven by GCMs, from the European ENSEMBLES Project (Christensen, Rummukainen, \& Lenderink, 2009) for the time period 1950-2050 under SRES A1B greenhouse gas and ozone recovery forcing scenarios are considered. The corresponding RCMs (driven by GCMs) are: C4IRCA3 (HadCM3Q16), CNRM/RM5.1 (ARPEGE RM5.1), DMI/ARPEGE (ARPEGE), DMI/BCM (BCM), DMI/ECHAM5-r3 (ECHAM5-r3), ETHZ/CLM (HadCM3Q0), METO_HC/HAD (HadCM3Q0), ICTP/REGCM3 (ECHAM5-r3), KNMI/RACMO2 (ECHAM5-r3), METNO/BCM (BCM), METNO/HadCM3Q0 (HadCM3Q0), MPIM/REMO (ECHAM5-r3), OURANOS/MRCC4.2.1 (CGCM3), SMHI/BCM (BCM), SMHI/ECHAM5-r3 (ECHAM5-r3), SMHI/HadCM3Q3 (HadCM3Q3), and UCLM/PROMES (HadCM3Q0).

Based on the grid provided by the RCMs dataset, 906 sites for analysis were set up (Figure 1). For the purposes of presentation and discussion of results, ten grid sites were selected for headwaters of main river basins (Figure 1): Galicia coast basin (site 80), Ebro River basin (140), Catalonian basin (251), Duero River basin (317), Tajo River basin (463), Guadiana River basin (554), Jucar River basin (644), Guadalquivir River basin (724), Segura 
River basin (759), and Andalusian Atlantic basins (799).

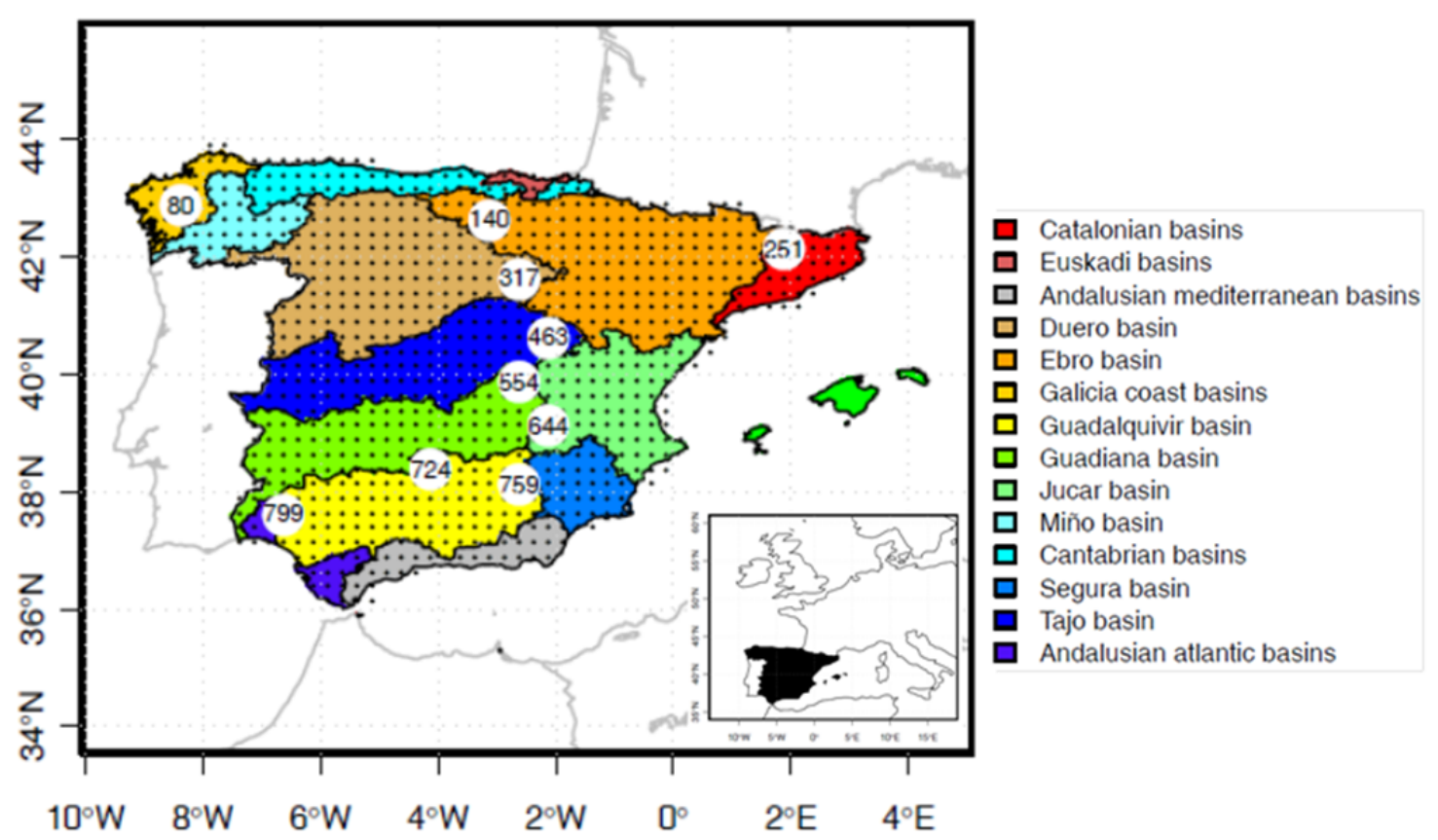

Figure 1. Study area with delineated basins, sites for modelling (906), and selected headbasins sites

\section{Methodology}

\subsection{Assessing Distributional Similarity between Observed Dataset and RCMs}

The annual maximum dry spells lengths series for the rainfall threshold $1 \mathrm{~mm} / \mathrm{d}$ were extracted for each site (according to Figure 1), from the datasets for the whole time period available (1950-2050). Then, the great variability exhibited by the time series of annual maximum dry spells lengths from RCMs is compared to that corresponding from Spain02 for 1961-1990 period, as represented by boxplots in Figure 2 for the sites selected. For example, for Segura (site 759) and Jucar River (644) basins, by visual inspection, the DMI/ARPEGE, ETHZ/CLM and KNMI/RACMO2 RCMs present the greater values of annual maximum dry spells lengths and more similarity (median, etc,) to those corresponding from the observed dataset.

The ability of RCMs models to capture annual maximum dry spells lengths in peninsular Spain was assessed by applying the Reliability Ensemble Average (REA) methodology developed by Giorgi \& Mearns (2002). The advantage of this methodology is the assessing of the ability of the RCMs (model reliability factor, $R$ ) to reproduce the present-day climate (the model performance criterion, $R_{B}$ ), while at the same time evaluating the convergence of different models to a given forcing scenario (by the convergence criterion, $R_{D}$ ), as follow:

$$
R=\left[\left(R_{B}\right)^{b} \times\left(R_{D}\right)^{d}\right]^{[1 /(b \times d)]}
$$

where the parameters $b$ and $d$ are the criterion weights. It is assumed $b=d=1$, giving equal weight to both criteria.

The estimation of $R_{B}$ and $R_{D}$ at grid site, was based on the p-value of the well known two-sample Smirnov-Kolmogorov goodness-of-fit test. In the case of the estimation of $R_{B}$, the CDFs were built from observed dataset and RCMs for 1961-1990. Figure 3 presents an example of CDFs for selected sites located in some headbasins. The results of visual inspection of Figure 2 related with sites 644 and 759 are therefore corroborated. 

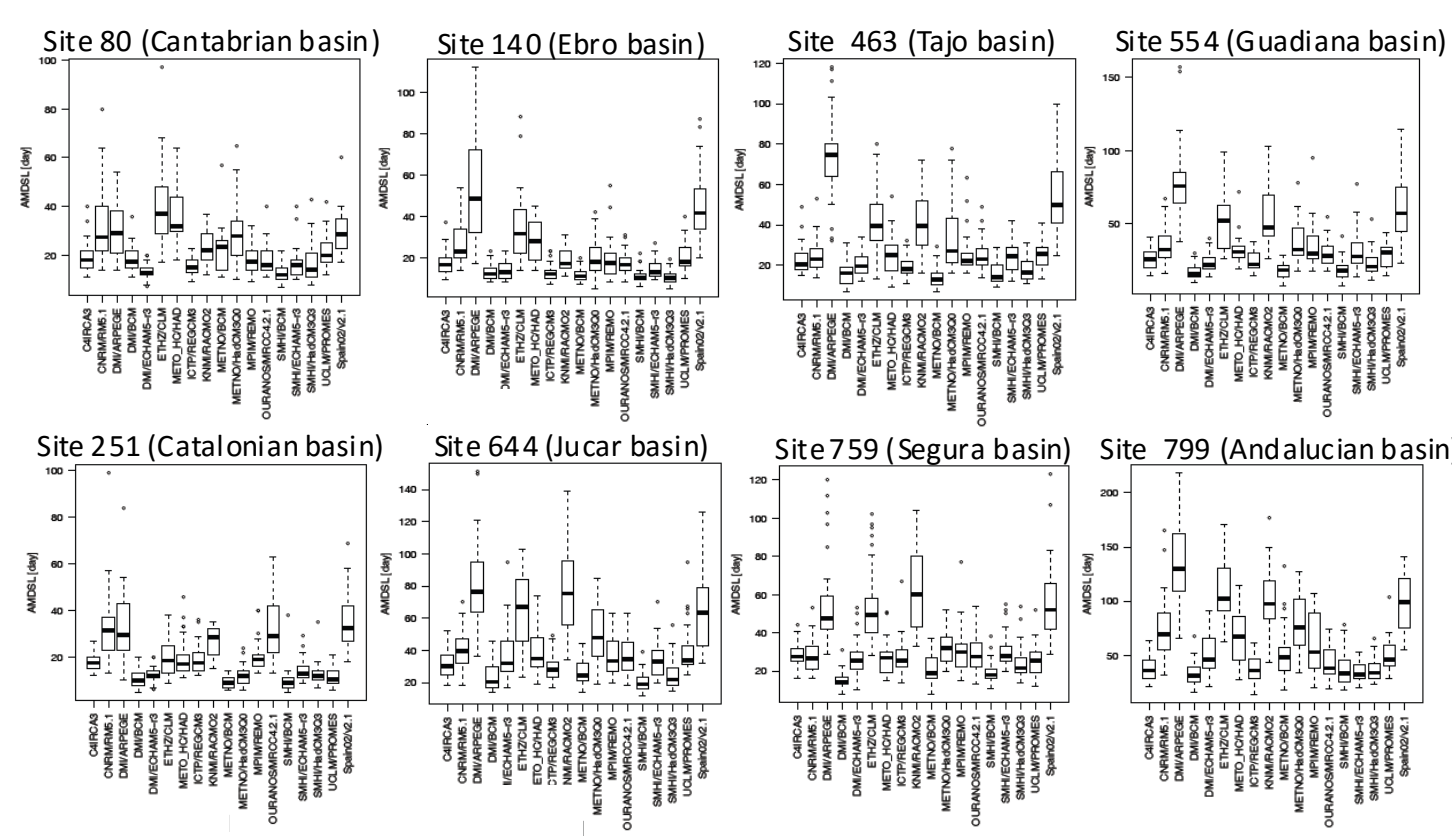

Figure 2. Boxplots of annual maximum dry spells lengths (AMDSL, days) for selected sites, from RCMs and observed dataset (1961-1990 period)
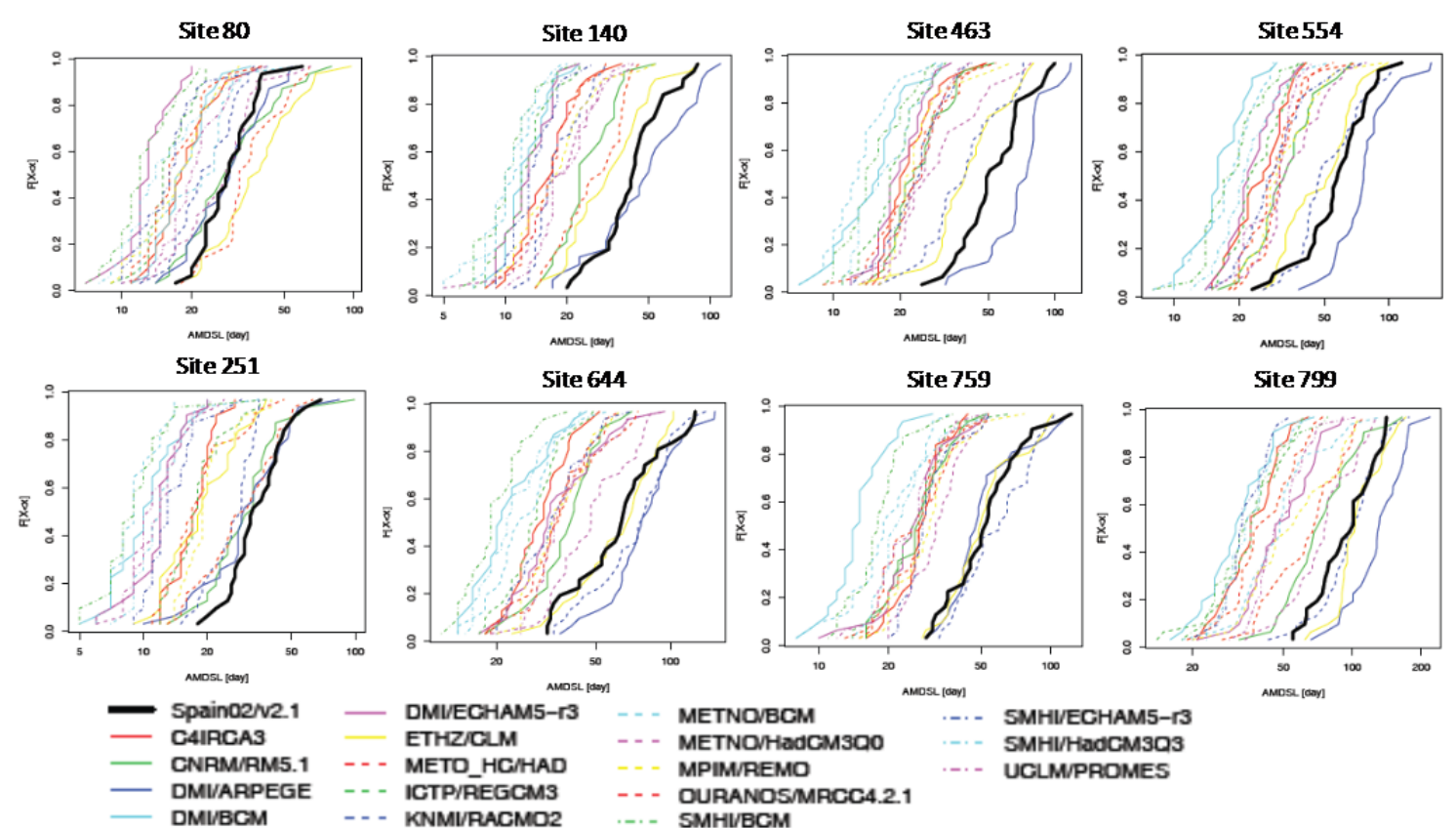

... - SMHIVEGHAM5-13 5MHI/HadCMsQ3 UGLM/PROMES

Figure 3. CDFs of annual maximum dry spells lenghts (AMDSL) from both observed dataset (in black) and RCMs (in colour), for 1961-1990 period

For $R_{D}$ estimation, an iterative process is followed to obtain the best estimated of the empirical PDF in the future using bootstrapping techniques on 2021-2050. Considering the values of $R_{B}$ and $R_{D}$ for each site (according to Figure 1), the maps of $R$ and the normalized reliability factor $\left(P_{m}\right)$ were obtained according to the REA method, as follow: 


$$
P m_{i}=\frac{R_{i}}{\sum_{1}^{N} R_{j}}
$$

\subsection{Building PDF Ensemble at Grid Site and Extreme Drought Hazard Maps}

The annual maximum dry spells lengths time series from RCMs were modelled by GAMLSS tools. GAMLSS consists of semi-parametric regression models, since they allow relating the parameters of a PDF as a function of an explanatory variable through non-parametric smoothing functions (Stasinopoulos \& Rigby, 2007). Several authors have applied GAMLSS for non-stationary modelling of extreme events (Villarini et al., 2009). In the present work, four PDFs of two parameters were considered for the GAMLSS application (Gumbel (GU), Gamma (GA), Lognormal (LN) and Weibull (WEI)). Then, the goodness-of-fit to the statistical model was assessed, considering the normality of the residuals, visual inspections of the qq-plots and the worm plot (not shown), following the methodology presented by van Buuren and Fredriks (2001).

To analyse plausible trends in annual maximum dry spells lengths, a PDF ensemble was built for each site. The skill score applied to each RCM was defined according to the performance in the REA analysis. Greater weight was given to RCMs with a high value of Pm using bootstrapping techniques.

Then the ensemble of PDF, with non-stationary parameters, was constructed for each ten years for the period 1961-2050. From the PDF ensemble, several statistics (mean, standard deviation, and annual maximum dry spells lengths for several $\mathrm{Tr}$ ) with their respective $95 \%$ confidence intervals (CI), could be estimated using bootstrapping techniques. Then, these values were interpolated to generate the spatio-temporal evolution of statistics. As an example, in Figure 4 the ensemble PDF in the selected sites of headbasins for 1990, 2020, and 2050 is presented with the $95 \%$ CI for 1990. Analysing Figure 4, on site 80 (Cantabrian basin), a significant change is observed for all quantiles for 2050, and not significant for 2020. Then, on site 644 (Jucar basin) and on site 759 (Segura basin), the changes are significant for low $\mathrm{Tr}$ in 2050. In general, an increase in annual maximum dry spells lengths is expected for 2020 and even higher for 2050 compared to 1990, significant in some areas. The exception is site 251 (Catalonian basin), where annual maximum dry spells lengths values are lower than expected for 2020. In the case of site 554 (Guadiana basin), the ensemble PDF for 2020 nearly coincides with the ensemble PDF for 1990, without significant changes.
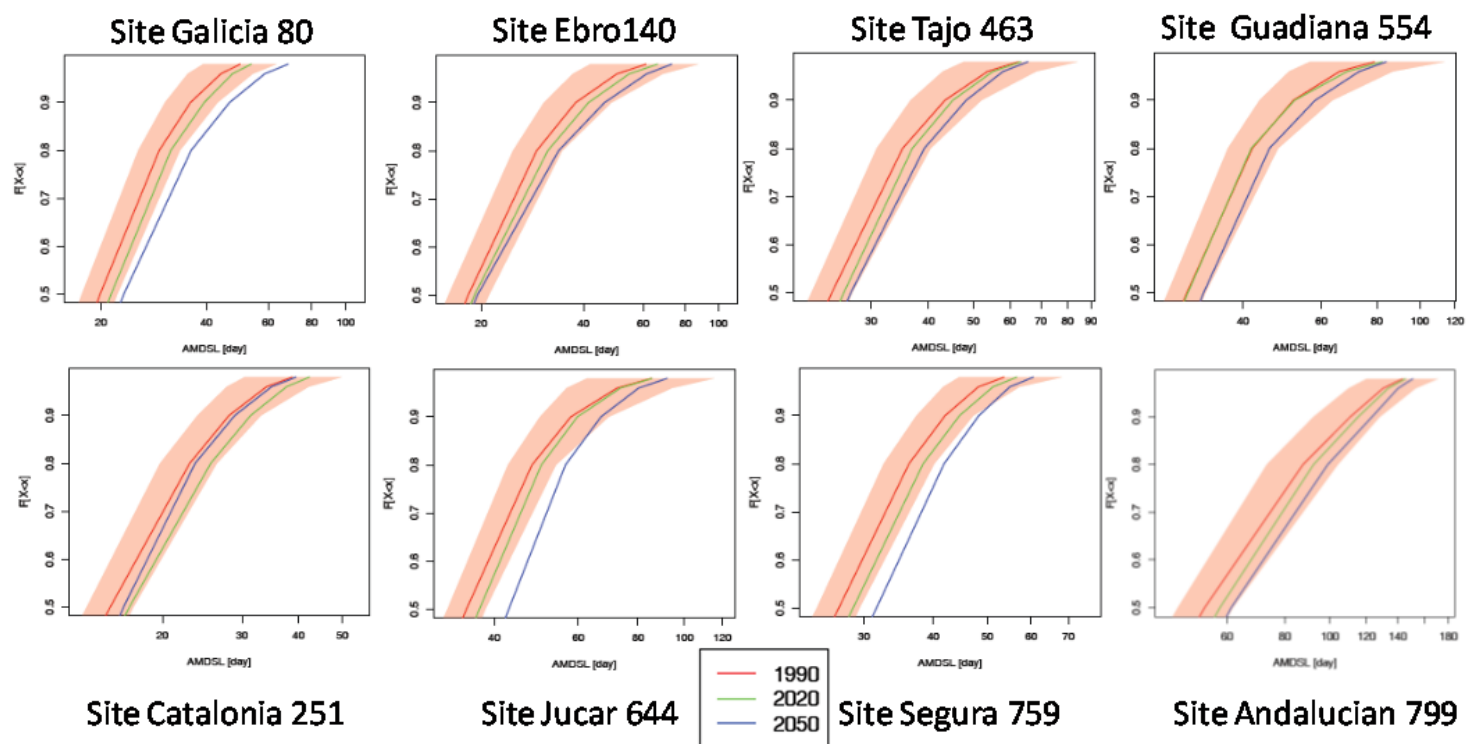

Site Catalonia 251

Site Jucar 644

- 2020 Site Segura 759

Site Andalucian 799

Figure 4. PDF ensemble in selected sites for 1990 (red); 2020 (green); and 2050 (blue); and confidence interval in solid (CI) for $95 \%$ for 1990 year reference

Figure 5 presents the maps constructed of annual maximum dry spells lengths for the $96 \%$ quantile $(\operatorname{Tr}=25 \mathrm{yrs}$. $)$ 
and $98 \%$ ( $T r=50$ yrs.) for the years 1990 and 2050, and their percentage difference. Using the Monte Carlo method based on bootstrapping with replacement, applied to annual maximum dry spells lengths populations obtained from PDF ensemble, the sites where these differences were statistically significant for $95 \%$ bootstrap CI have been shown on difference maps with a dark grey square (Figure 5). The significantly longer dry spells, especially for 2050, are observed in basins of the South-East such as the Jucar basin, Segura basin, Andalusian basins, lower Guadalquivir basin, and Guadiana basin. The change maps show a general increase for both $\operatorname{Tr}$ throughout Spain. The spatial distributions exhibit significant changes of annual maximum dry spells lengths $96 \%$ with intensification of droughts events with increases of about 30-40 \% in Galicia (mainly Miño basin), but also includes wide areas of Andalusia, the lower-medium Duero and Tajo basin, and about $20 \%$ for North-East of the Iberian Peninsula (medium Ebro basin) and for the Segura basin, as happened with the maps of standard deviation change (not shown). Then, for annual maximum dry spells lengths $98 \%$, the difference map exhibits greater values but fewer zones with significant values.
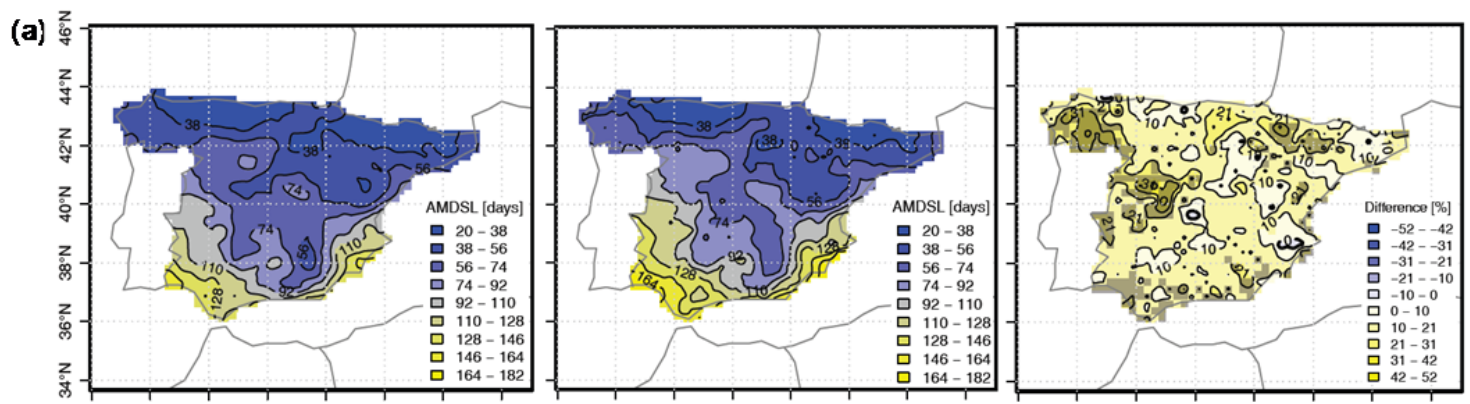

(b)
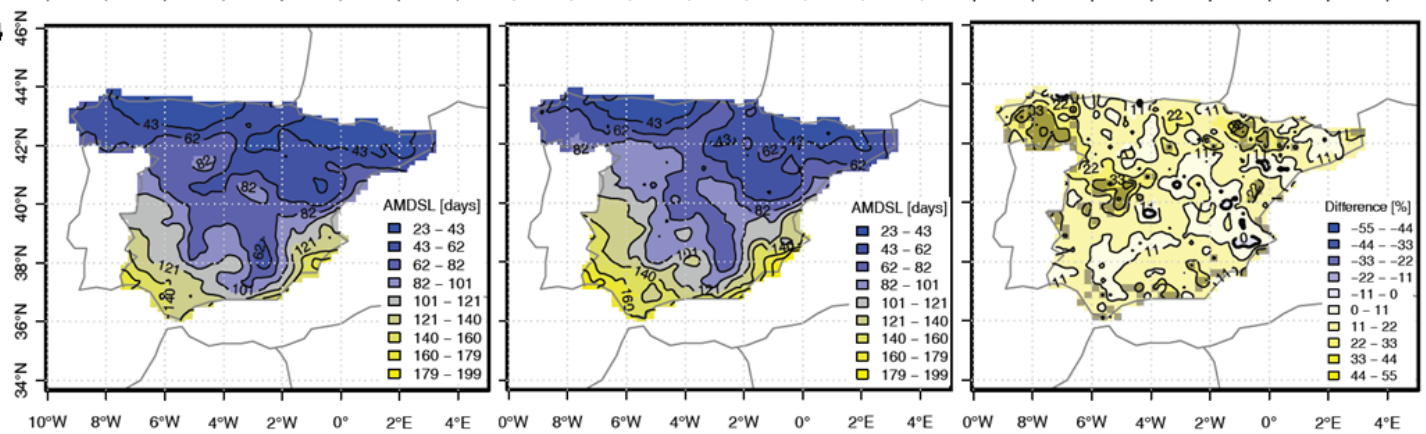

Figure 5. Maps of annual maximum dry spells lenghts for 1990 in first column, 2050 in central column, and their percentage change (\%) assessed as [100×(map2050-map1990)/map1990] in last column: (a) $\mathrm{Tr}=25$ yrs., and (b) $\operatorname{Tr}=50$ yrs. The shaded areas represent significant change/difference ( $95 \%$ confidence interval)

\section{Discussion and Conclusions}

A This study describes the variability and discontinuities detected in the spatial patterns of annual extreme dry spells in peninsular Spain, by non-stationary modelling and ensemble techniques of RCMs. Improving the knowledge regarding expected trends of annual maximum dry spells lenghts at basin scale, contributes to designing more effective drought contingency preparedness and recovery operations.

To take into account the variability over time in drought hazard, time-dependent parameters of the PDFs are estimated by GAMLSS tools. High performing RCMs in the simulation of annual maximum dry spells lenghts were KNMI/RACMO2, MPIM/REMO, and OURANOS/MRCCC4.2.1 RCMs for South-East Spain. UCLM/PROMES and DMI/ECHAM5-r3 RCMS are highlighted in the North and North-East of Spain; and MPIM/REMO RCMs in the central zone of peninsular Spain. In order to exploit all the information provided by the RCMs available, a PDF ensemble is constructed at grid site based on the distributional similarity and bootstrapping techniques, via REA methodology. The extreme droughts maps associated with $T r=25$ and 50 yrs., from the PDF ensemble, present an appreciable general increase in the spatial pattern for the horizon 2050 in contrast with 1990. While trends in precipitation may not translate directly into changes in streamflows, the intensification of droughts obviously has an impact on the various components of the water cycle.

In conclusion, a methodology is presented for assessing the impacts of climate change on spatio-temporal 
distributions of extreme drought, identifying the more prone areas associated with $T r$.

\section{Acknowledgments}

The work was supported by the R\&D National Project Grant No. CGL2012-39895-C02-01, financed by the National Secretary of Research of Spanish Ministry of Science and Innovation and FEDER funds. The funding received from Spanish Ministry of Education, Culture and Sport for Mobility Grant of Senior Professors and Researchers (Grant No. PRX14/00748), is gratefully acknowledged.

\section{References}

Bonaccorso, A. B., Bordi, I., Cancelliere, A., Rossi, G., \& Sutera, A. (2003). Spatial variability of drought: An analysis of the SPI in Sicily. Water Resources Management, 17(4), 273-296. http://dx.doi.org/10.1023/A:1024716530289

Christensen, J. H., Rummukainen, M., \& Lenderink, J. F. B. (2009). Formulation of very-high-resolution regional climate model ensembles for Europe. In P. Van Der Linden, \& J. F. B. Mitchell (Eds.), Climate Change and its Impacts: Summary of Research and Results from the ENSEMBLES project. Met Office Hadley Centre, Exeter, UK.

EEA (European Environment Agency). (2010). The European Environment, State and outlook 2010: water resources: quantity and flows. Copenhagen Denmark, European Environment Agency. http://dx.doi.org/10.2800/59600

Efron, B., \& Tibshirani, R. J. (1993). An Introduction to the Bootstrap. Chapman \& Hall, New York, USA.

García Galiano, S. G., \& Giraldo Osorio, J. D. (2010). Analysis of impacts on hydrometeorological extremes in the Senegal River Basin from REMO RCM. Met. Z., 19(4), 375-384. http://dx.doi.org/10.1127/0941-2948/2010/0457

Giorgi, F., \& Mearns, L. O. (2002). Calculation of average, uncertainty range, and reliability of regional climate changes from AOGCM simulations via the "reliability ensemble averaging" (REA) method. J. Climate, 15(10), 1141-1158. http://dx.doi.org/10.1175/1520-0442(2002)015<1141:COAURA>

Herrera, S., Fita, L., Fernández, J., \& Gutiérrez, J. M. (2010). Evaluation of the mean and extreme precipitation regimes from the ENSEMBLES regional climate multimodel simulations over Spain. J. Geophysical Research, 115. http://dx.doi.org/10.1029/2010JD013936

Milly, P. C. D., et al. (2008). Stationarity is dead: Whiter water management? Science, 319, 573-574. $\mathrm{http}: / / \mathrm{dx}$. doi.org/10.1126/science.1151915

Raziei, T., Saghafian, B., Paulo, A. A., Pereira, L. S., \& Bordi, I. (2008). Spatial patterns and temporal variability of drought in Western Iran. Water Resour Manage., 23(3), 439-455. http://dx.doi.org/10.1007/s11269-008-9282-4

Rigby, R. A., \& Stasinopoulos, D. M. (2005). Generalized additive models for location, scale and shape. Appl. Stat., 54, 507-554. http://dx.doi.org/0035-9254/05/54507

Ruíz Sinoga, J. D., García Marín, R., Martínez Murillo, J. F., \& Gabarron Galeote, M. A. (2011). Precipitation dynamics in southern Spain: trends and cycles. International Journal of Climatology, 31, 2281-2289. http://dx.doi.org/10.1002/joc.2235

Sánchez, E., Romera, R., Gaertner, M. A., Gallardo, C., \& Castro, M. (2009). A weighting proposal for an ensemble of regional climate models over Europe driven by 1961-2000 ERA40 based on monthly precipitation probability density functions. Atmos. Sci. Let., 10(4), 241-248. http://dx.doi.org/10.1002/asl.230

Stasinopoulos, D. M., \&Rigby, R. A. (2007). Generalized additive models for location, scale and shape (GAMLSS) in R. J. Stat. Software, 23(7), 1-46. http://dx.doi.org/10.18637/jss.v023.i07

Van Buuren, S., \& Fredriks, M. (2001). Worm plot: a simple diagnostic device for modelling growth reference curves. Statist. Med., 20, 1259-1277. http://dx.doi.org/10.1002/sim.746

Vasiliades, L., Loukas, A., \& Liberis, N. (2011). A water balance derived drought index for Pinios River Basin, Greece. Water Resour Manage, 25(4), 1087-1101. http://dx.doi.org/10.1007/s11269-010-9665-1

Vicente Serrano, S. M., \& Beguería Portugues, S. (2003). Estimating extreme dry-spell risk in the middle Ebro valley (northeastern Spain): a comparative analysis of partial duration series with general Pareto distribution and annual maxima series with Gumbel distribution. Int. J. Climatol., 23, 1103-1118. 
http://dx.doi.org/10.1002/joc.934

Villarini, G., Smith, J. A., Serinaldi, F., Bales, J., Bates, P. D., \& Krajewski, W. F. (2009). Flood frequency analysis for nonstationary annual peak records in a urban drainage basin. Adv. Water Resour., 32, 1255-1266. http://dx.doi.org/10.1016/j.advwatres.2009.05.003

\section{Copyrights}

Copyright for this article is retained by the author(s), with first publication rights granted to the journal.

This is an open-access article distributed under the terms and conditions of the Creative Commons Attribution license (http://creativecommons.org/licenses/by/3.0/). 